\title{
Sentinel lymph node biopsy in breast cancer patients undergoing skin/nipple-sparing mastectomy and immediate autologous reconstruction
}

\author{
SATORU TANAKA, TAKEHIRO NOHARA, MITSUHIKO IWAMOTO, KAZUHIRO SUMIYOSHI, \\ KOSEI KIMURA, YUKO TAKAHASHI and NOBUHIKO TANIGAWA
}

Department of General and Gastroenterological Surgery, Osaka Medical College, Takatsuki City, Osaka 569-8686, Japan

Received July 22, 2009; Accepted October 5, 2009

DOI: 10.3892/ol_00000018

\begin{abstract}
Immediate breast reconstruction following mastectomy is an effective treatment for breast cancer patients. Among several implant options, a latissimus dorsi myocutaneous (LDM) flap is used mainly due to the ease and minimal invasiveness of the procedure. The role of sentinel lymph node (SLN) biopsy with total mastectomy is evolving. Since SLN biopsy is not included in health insurance coverage in the treatment of patients in Japan, it is not generally performed as a separate procedure due to its cost. The present study reviewed the results of seven patients who underwent initialstaged SLN biopsy followed by planned mastectomy and LDM flap reconstruction. Two patients with positive SLNs showed macrometastases and underwent modified radical mastectomy with immediate reconstruction. In contrast, cases showing negative results for sentinel lymph nodes underwent total mastectomy. There were no false-negative cases among the SLN biopsy-negative cases. When an SLN is found to be positive on final pathology, the patient with reconstruction by LDM flap generally requires a potentially difficult reoperation on the remaining axillary nodes. When initial SLN biopsy is generally performed as a separate procedure in Japan, it will be an effective method for screening the axilla for patients who wish to undergo LDM flap reconstruction.
\end{abstract}

\section{Introduction}

Immediate breast reconstruction following mastectomy has been used frequently as an effective treatment option for patients with early-stage breast cancer. Current techniques focus on a less radical removal of skin, such as skin-sparing

Correspondence to: Dr Nobuhiko Tanigawa, Department of General and Gastroenterological Surgery, Osaka Medical College, 2-7 Daigaku-machi, Takatsuki City, Osaka 569-8686, Japan

E-mail: sur001@poh.osaka-med.ac.jp

Key words: sentinel lymph node biopsy, immediate breast reconstruction, skin-sparing mastectomy, nipple-sparing mastectomy mastectomy (SSM) (1-3), often performed only through a circumareolar keyhole incision (4), or nipple-sparing mastectomy (NSM) (5). Cosmetically, these techniques are extremely effective, as the greater part of the native skin and inframammary fold of the breast are conserved (6). Among the various immediate breast reconstruction methods available, autologous tissue has been accepted as the standard option. Regarding the implant, a latissimus dorsi myocutaneous (LDM) flap is typically used in cases with small breast volume or in women hoping to have a baby, due to the ease and minimal invasiveness of the procedure.

Sentinel lymph node (SLN) biopsy is a common procedure for tumor staging. Furthermore, several studies have validated the effectiveness and safety of the procedure in node-negative patients with no increase in local axillary recurrence or distal metastatic spread when compared to axillary lymph node dissection (7-10). However, SLN biopsy is not performed in all institutions since it has not been included in health insurance coverage in Japan. Nodal involvement is classified into three categories: macrometastasis (diameter $>2 \mathrm{~mm}$ ), micrometastasis (diameter 0.2-2.0 mm) and isolated tumor cells (ITCs) (diameter $<0.2 \mathrm{~mm}$ ). Macrometastases certainly have an effect on patient treatment and should obviously be considered clinically relevant. ITCs are regarded as non-significant in terms of staging and treatment. Conversely, the significance of micrometastases remains unknown, with different studies sometimes reaching contradictory conclusions (11).

Macrometastases can be identified by an intraoperative examination of histological sections at intervals of $2 \mathrm{~mm}$, but such sections may reduce the likelihood of identifying micrometastases. Axillary lymph node dissection (ALND) has been recommended in cases with positive SLNs $(12,13)$, but ALND may be avoided in cases of micrometastases given the risks associated with ALND, including lymphedema, limitations to arm mobility and sensory loss (14-16). Conversely, in patients with immediate autologous flap, particularly LDM flap reconstruction, secondary ALND, following identification of a positive SLN upon permanent histological examination after the initial operation is considered a more difficult procedure. Secondary ALND is a difficult procedure because either reoperation of the reconstructed breast or second surgery through the axillary incision is required, both of which may compro- 
Table I. Clinical characteristics of the seven cases.

\begin{tabular}{lcccccc}
\hline Case & Age (years) & Tumor size & Histological type & No. of SLNs & SLN status & Treatment \\
\hline 1 & 74 & T2 & IDC & 2 & Positive (1/2) & MRM with LDM \\
2 & 29 & T1 & IDC & 3 & Negative & TM with LDM \\
3 & 45 & Tis & DCIS & 3 & Negative & TM with LDM \\
4 & 50 & Tis & DCIS & 2 & Negative & TM with LDM \\
5 & 41 & T2 & IDC & 3 & Positive (1/3) & MRM with LDM \\
6 & 38 & Tis & DCIS & 2 & Negative & TM with LDM \\
7 & 43 & T2 & IDC & 2 & Negative & TM with LDM \\
\hline
\end{tabular}

Tis, carcinoma in situ; $\mathrm{T} 1$, tumor $\leq 2 \mathrm{~cm}$ in greatest dimension; T2, tumor $>2 \mathrm{~cm}$ but not $>5 \mathrm{~cm}$ in greatest dimension; IDC, invasive ductal carcinoma; DCIS, ductal carcinoma in situ; SLN, sentinel lymph node; TM, total mastectomy; MRM, modified radical mastectomy; LDM, latissimus dorsi myocutaneous flap.

mise the blood supply of the pedicle or negatively affect the aesthetic results (17-19).

We performed immediate LDM flap following NSM/SSM with SLN biopsy for early-stage breast cancer patients. The present study reviewed the results for patients who underwent SLN biopsy before definitive mastectomy and reconstruction.

\section{Materials and methods}

Between April 2007 and March 2009, seven patients with early breast cancer and a clinically negative axilla underwent SSM/NSM with immediate LDM flap reconstruction and SLN biopsy. No patients were treated with preoperative chemotherapy or hormonal therapy. Breast cancer was diagnosed based on the results of a core needle biopsy. In all cases, SLN biopsy was performed using a combination technique involving the technetium $99 \mathrm{~m}$ tin colloid $(74 \mathrm{MBq})$ and indigo blue dye, which were injected intracutaneously into the areola. Intraoperatively, sentinel lymph nodes were evaluated by $2-\mathrm{mm}$ serial sections stained with hematoxylin and eosin. Pathological evaluation with permanent sections was performed using hematoxylin and eosin with added immunohistochemical staining in cases of suspected metastasis.

\section{Results}

Patient and tumor characteristics are summarized in Table I. SLN biopsy was performed for 158 breast cancer patients at our institution over the defined time period. Seven of these patients were planning mastectomy with immediate reconstruction using LDM flap and were managed with an initial SLN biopsy. Tumor types included large ductal carcinoma in situ (DCIS) $(\mathrm{n}=3)$ and invasive ductal carcinoma $(\mathrm{n}=4)$. DCIS cases included one high-grade DCIS. There were two patients with $\mathrm{T} 1$ and two patients with $\mathrm{T} 2$ primary lesions, respectively. Primary SLN dissection yielded 17 nodes in seven patients. Positive SLNs were identified in two patients, representing macrometastases (diameter $>2 \mathrm{~mm}$ ) in both cases. Patients with negative results for the SLN biopsy underwent total mastectomy followed by immediate reconstruction. In contrast, patients with positive results for the SLN biopsy underwent modified radical mastectomy with immediate reconstruction. The patients were reconstructed using an LDM flap. In the two cases with positive SLNs, one patient had no metastases in non-sentinel nodes and the other had metastases. No patients with negative results from the SLN biopsy revealed tumor metastases in permanent sections (no false-negatives). Patients with metastases identified in SLNs underwent adjuvant chemotherapy.

\section{Discussion}

SSM/NSM followed by immediate breast reconstruction is one approach aiming to achieve the best possible cosmetic result in one procedure and one hospital stay and to minimize the physiological and physical trauma of breast cancer. Moreover, with the advent of SLN biopsy techniques that decrease the risk of complications of ALND, the current approach to breast surgery aims to further minimize tissue loss while obtaining maximal oncological safety. The present study reviewed our experience with SLN biopsy before SSM/NSM followed by immediate LDM flap.

Although SLN biopsy is now standard procedure, it is not performed as a separate procedure due to its cost in all Japanese institutions. In most institutions, intraoperative analysis of the SLN status proceeds to ALND immediately on identification of a positive node. Even though macrometastases can be identified by $2-\mathrm{mm}$ serial sections intraoperatively, micrometastases may be overlooked. The current standard of care is to recommend secondary ALND in patients with positive SLNs which have been identified by a permanent histological examination $(12,13)$. ALND is not always required for cases of micrometastases overlooked by intraoperative examination (14-16), given the significantly decreased risk of finding positive non-SLNs in patients with SLN micrometastases compared with SLN macrometastases and given the unclear local control and prognostic impact between ALND and adjuvant systemic therapy for micrometastases (20-23).

For patients considering total mastectomy with immediate reconstruction, the option of SLN biopsy may create unique problems. Numerous plastic surgical techniques are available for breast reconstruction. Tissue expander with revision to permanent implant is considered the least complex option. Despite the availability of this option, return to the axilla 
following placement of a synthetic device risks implant damage and infection, necessitating replacement (24). Placement of an expander represents the lowest risk for subsequent return to surgery. The transverse rectus abdominis myocutaneous flap is more technically demanding, but provides autologous tissue replacement of absent breast parenchyma. This procedure requires displacement of abdominal muscle and vascular tissue into the chest area. Using a pedicled approach, the axilla is relatively spared, and return to the axilla is technically feasible. The LDM flap is becoming increasingly popular as a reconstructive technique. This technique involves transposition of muscle from the back to the anterior chest wall and medial fixation near the sternum, preserving native nerve and blood supply $(25,26)$. This may represent the most difficult surgical environment for a return to the axilla, since a reversal of the flap is required during ALND. Fortunately, we encountered no false-negative cases in this study. However, we do not recommend that patients with false-negative results undergo further ALND. Althernative types of therapy such as systemic or radiation are suggested at our institution due to our limited knowledge of the prognostic significance of ALND, complications after ALND and the difficulty of the surgical procedure. When the significance of ALND in cases of micrometastases are clarified by currently ongoing randomized clinical trials of SLN biopsy $(27,28)$, we suggest initial SLN biopsy as a separate procedure in a day surgery under local anesthesia in order to determine whether the appropriate surgery should be modified radical mastectomy or total mastectomy with immediate LDM flap reconstruction. In addition, when breast cancer patients are entitled to SLN biopsy under health insurance coverage in Japan, initial SLN biopsy as a separate procedure will offer a more general approach.

\section{References}

1. Slavin SA, Schnitt SJ, Duda RB, et al: Skin-sparing mastectomy and immediate reconstruction: oncologic risks and aesthetic results in patients with early-stage breast cancer. Plast Reconstr Surg 102: 49-62, 1998.

2. Kroll SS, Khoo A, Singletary SE, et al: Local recurrence risk after skin-sparing and conventional mastectomy: a 6-year followup. Plast Reconstr Surg 104: 421-425, 1999.

3. Carlson GW, Losken A, Moore B, Thornton J, Elliott M, Bolitho G and Denson DD: Results of immediate breast reconstruction after skin-sparing mastectomy. Ann Plast Surg 46: 222-228, 2001.

4. Gabka CJ, Maiwald G and Bohmert H: Immediate breast reconstruction for breast carcinoma using the periareolar approach. Plast Reconstr Surg 101: 1228-1234, 1998.

5. Gerber B, Krause A, Reimer T, et al: Skin-sparing mastectomy with conservation of the nipple-areola complex and autologous reconstruction is an oncologically safe procedure. Ann Surg 238: 120-127, 2003.

6. Salhab M, Al Sarakbi W, Joseph A, Sheards S, Travers J and Mokbel K: Skin-sparing mastectomy and immediate breast reconstruction: patient satisfaction and clinical outcome. Int J Clin Oncol 11: 51-54, 2006.

7. Veronesi U, Paganelli G, Viale G, et al: A randomized comparison of sentinel-node biopsy with routine axillary dissection in breast cancer. N Engl J Med 349: 546-553, 2003.

8. Roumen RM, Kuijt GP, Liem IH and van Beek MW: Treatment of 100 patients with sentinel node-negative breast cancer without further axillary dissection. Br J Surg 88: 1639-1643, 2001.
9. Schrenk P, Hatzl-Griesenhofer M, Shamiyeh A and Waynad W: Follow-up of sentinel node negative breast cancer patients without axillary lymph node dissection. J Surg Oncol 77: 165-170, 2001.

10. Hansen NM, Grube BJ and Giuliano AE: The time has come to change the algorithm for the surgical management of early breast cancer. Arch Surg 137: 1131-1135, 2002.

11. Dowlatshahi K, Fan M, Snider HC and Habib FA: Lymph node micrometastases from breast carcinoma: reviewing the dilemma. Cancer 80: 1188-1197, 1997.

12. Reynolds C, Mick R, Donohue JH, et al: Sentinel lymph node biopsy with metastasis: can axillary dissection be avoided in some patients with breast cancer? J Clin Oncol 17: 1720-1726, 1999.

13. Rahusen FD, Torrenga H, van Diest PJ, Pijpers R, van der Wall E, Licht $\mathbf{J}$ and Meijer S: Predictive factors for metastatic involvement of nonsentinel nodes in patients with breast cancer. Arch Surg 136: 1059-1063, 2001.

14. Fournier K, Schiller A, Perry RR and Laronga C: Micrometastasis in the sentinel lymph node of breast cancer does not mandate completion axillary dissection. Ann Surg 239: 859-865, 2004.

15. Rutledge H, Davis J, Chiu R, Cibull M, Brill Y, McGrath P and Samayoa L: Sentinel node micrometastasis in breast carcinoma may not be an indication for complete axillary dissection. Mod Pathol 18: 762-768, 2005.

16. Rutgers EJ: Sentinel node biopsy: interpretation and management of patients with immunohistochemistry-positive sentinel nodes and those with micrometastases. J Clin Oncol 26: 698-702, 2008.

17. Stradling BL, Ahn M, Angelats J and Gabram SG: Skin-sparing mastectomy with sentinel lymph node dissection: less is more. Arch Surg 136: 1069-1075, 2001.

18. Choi SH, Barsky SH and Chang HR: Clinicopathologic analysis of sentinel lymph node mapping in early breast cancer. Breast $\mathrm{J}$ 9: 153-162, 2003.

19. Kronowitz SJ, Chang DW, Robb GL, et al: Implications of axillary sentinel lymph node biopsy in immediate autologous breast reconstruction. Plast Reconstr Surg 109: 1888-1896, 2002.

20. Chu KU, Turner RR, Hansen NM, Brennan MB, Bilchik A and Giuliano AE: Do all patients with sentinel node metastasis from breast carcinoma need complete axillary node dissection? Ann Surg 229: 536-541, 1999.

21. Weiser MR, Montgomery LL, Tan LK, Susnik B, Leung DY, Borgen PI and Cody HS III: Lymphovascular invasion enhances the prediction of non-sentinel node metastases in breast cancer patients with positive sentinel nodes. Ann Surg Oncol 8: 145-149, 2001.

22. Den Bakker MA, van Weeszenberg A, de Kanter AY, Beverdam FH, Pritchard C, van der Kwast TH and MenkePluymers M: Non-sentinel lymph node involvement in patients with breast cancer and sentinel node micrometastasis; too early to abandon axillary clearance. J Clin Pathol 55: 932-935, 2002.

23. Jakub JW, Diaz NM, Ebert MD, et al: Completion axillary lymph node dissection minimizes the likelihood of false negatives for patients with invasive breast carcinoma and cytokeratin positive only sentinel lymph nodes. Am J Surg 184: 302-306, 2002.

24. Fine N, Mustoe T and Fenner G: Breast reconstruction. In: Disease of the Breast. Harris J, Lippman M, Morrow M and Osborne C (eds). Lippincott Williams \& Wilkins, Phildelphia, pp561-575, 2000.

25. Delay E, Gounot N, Bouillot A, Zlatoff P and Rivoire M: Autologous latissimus breast reconstruction: a 3-year clinical experience with 100 patients. Plast Reconstr Surg 102: 1461-1478, 1998.

26. Papp C and McCraw JB: Autogenous latissimus breast reconstruction. Clin Plast Surg 25: 261-266, 1998.

27. Leitch AM, Beitsch PD, McCall LM, et al: Patterns of participation and successful patient recruitment to American College of Surgeons Oncology Group Z0010, a phase II trial for patients with early-stage breast cancer. Am J Surg 190: 539-542, 2005.

28. http://www.ibcsg.org/Pages/default.aspx. 